\title{
Digitalisierung für alle? Zur Auswirkung digitaler Angebote auf Teilhabechancen von Wohnungslosen
}

\author{
Frank Sowa • Benedikt Rösch • Tanja Holzmeyer • Marcel Neberich • \\ Frank Opferkuch • Katrin Proschek • Richard Reindl • Joachim Scheja • \\ Sigrid Zauter
}

Online publiziert: 18. März 2020

(C) Der/die Autor(en) 2020

Zusammenfassung Das an der Technischen Hochschule Nürnberg Georg Simon Ohm angesiedelte Forschungsprojekt „SIWo - Smart Inklusion für Wohnungslose“ widmet sich der Fragestellung, welche Informations-, Unterstützungs- und Beratungsbedarfe bei wohnungslosen Menschen vorliegen und in welcher Form sich die Teilhabechancen für die heterogene Zielgruppe wohnungsloser oder von Wohnungsnot betroffener Menschen mittels digitaler Hilfe und Beratungsangebote verbessern lassen. Hierfür werden zum einen mit Gruppendiskussionen und qualitativen Interviews Bedarfe identifiziert. Zum anderen sollen im Rahmen des Projekts prototypische Lösungsansätze in Form einer plattformübergreifenden Hilfe-App entwickelt, getestet und mit Evaluationsverfahren begleitet werden. Charakteristisch ist dabei die enge interdisziplinäre Zusammenarbeit zwischen Sozialwissenschaft, Informatik

Prof. Dr. F. Sowa $(\bowtie) \cdot$ Prof. Dr. R. Reindl

Fakultät Sozialwissenschaften, Technische Hochschule Nürnberg Georg Simon Ohm,

90121 Nürnberg, Deutschland

E-Mail: frank.sowa@th-nuernberg.de

Prof. Dr. R. Reindl

E-Mail: richard.reindl@th-nuernberg.de

B. Rösch · T. Holzmeyer $\cdot$ K. Proschek $\cdot$ S. Zauter

Institut für E-Beratung, Technische Hochschule Nürnberg Georg Simon Ohm, 90121 Nürnberg,

Deutschland

B. Rösch

E-Mail: benedikt.roesch@th-nuernberg.de

T. Holzmeyer

E-Mail: tanja.holzmeyer@th-nuernberg.de

K. Proschek

E-Mail: katrin.proschek@th-nuernberg.de

S. Zauter

E-Mail: sigrid.zauter@th-nuernberg.de 
und Energietechnik, die partizipative Begleitung des Projekts durch Wohnungslose und andere Stadtakteur*innen, sowie der Fokus auf interaktive Funktionen, die digitalisierte Begegnungsräume ermöglichen und Interaktionen auch zwischen Bevölkerungsgruppen fördern, die i.d. R. eher wenig miteinander zu tun haben. Dieses Vorgehen begreift somit Wohnungslosigkeit nicht ausschließlich als professionelles Arbeitsfeld der Sozialen Arbeit, sondern als gesamtgesellschaftliche Aufgabe.

Schlüsselwörter Wohnungslosigkeit · Vulnerabilität · Bedarfe · Soziale Arbeit · Digitalisierung

\title{
Digitization for Everybody? The impact of digital offers on opportunities for homeless people to participate
}

\begin{abstract}
The research project "SIWo-Smart Inklusion für Wohnungslose" (Smart Inclusivity for homeless people) of Nuremberg Tech Georg Simon Ohm addresses the question which needs for information, support and counselling homeless people have and in what way equal access for this diverse target group, homeless people and people affected by housing shortage, can be supported by digital help and counselling solutions. For this purpose, on the one hand needs will be identified by group discussions and qualitative interviews. On the other hand, development, test and socio-scientific evaluation of prototypical solutions for a platform independent help-app is planned. Specific for the approach is close interdisciplinary collaboration between social sciences, computer sciences and power engineering as well as the participation of homeless people and other city community stakeholders. Focus is on interactive functionalities which enable digitally supported contact and interaction between different public groups who wouldn't regularly interact. This approach understands homelessness not exclusively as a professional field of social work but as a challenge for society as a whole.
\end{abstract}

Keywords Homelessness $\cdot$ Vulnerability $\cdot$ Needs $\cdot$ Social work $\cdot$ Digitization

\footnotetext{
M. Neberich · Prof. Dr. F. Opferkuch

Fakultät Elektrotechnik, Feinwerktechnik, Informationstechnik, Technische Hochschule Nürnberg Georg Simon Ohm, 90121 Nürnberg, Deutschland

M. Neberich

E-Mail: m.neberich@th-nuernberg.de

Prof. Dr. F. Opferkuch

E-Mail: frank.opferkuch@th-nuernberg.de

Prof. Dr. J. Scheja

Fakultät Informatik, Technische Hochschule Nürnberg Georg Simon Ohm, 90121 Nürnberg,

Deutschland

E-Mail: joachim.scheja@th-nuernberg.de
} 


\section{Problemstellung und Forschungsstand}

Obwohl die Wege in die Wohnungs- und Obdachlosigkeit aufgrund des jeweiligen biographischen Hintergrunds und der Problemlagen der Betroffenen sehr vielfältig und vielschichtig sind, dominiert bei den meisten eine prekäre finanzielle Lage bzw. materielle Armut (Gerull 2014; Malyssek und Störch 2009; Paulgerg-Muschiol 2009; Levinson 2004). Diese geht einher mit einem weitreichenden Verlust von materiellen, kulturellen, sozialen und politischen Möglichkeiten am Leben der Gesellschaft teilzunehmen. Dazu zählen beispielsweise eine Benachteiligung bei der institutionellen Versorgung mit Gütern und Dienstleistungen, der Ausschluss von der Nutzung der regulären technisch-materiellen urbanen Infrastruktur, der Verlust des Ansehens, negative Etikettierungen und Stigmatisierung. Des Weiteren besteht die Gefahr der Verengung der sozialen Kontakte auf ein Milieu mit geringer Reichweite und die Gefahr der sozialen Isolation durch eine Reduzierung der sozialen Kontakte. Insgesamt ist festzustellen, dass die Lebenslagen von wohnungslosen Personen häufig durch Multiproblemlagen gekennzeichnet sind, also eine Mehrfachbelastung durch Faktoren wie z.B. Armut, Wohnungslosigkeit, Suchtproblematik, psychische und/oder somatische Erkrankungen, Arbeitslosigkeit und Überschuldung aufweisen (Paegelow 2012). Von fehlenden Teilhabemöglichkeiten sind jedoch nicht nur wohnungslose und obdachlose Menschen betroffen, vielmehr weitet sich in Zeiten der Verknappung von leistbarem Wohnraum der Kreis derjenigen, die vom Wohnraumverlust bedroht sind, auf immer weitere Bevölkerungsgruppen aus (Sowa 2020; Holm 2014; Wehrheim 2012). Damit wird die ohnehin schon heterogene Gruppe der Wohnungslosen noch heterogener.

Bisherige gesellschaftliche Teilhabebemühungen delegieren das soziale Problem der Wohnungslosigkeit auf die Angebote Sozialer Arbeit in der Wohnungslosenhilfe, welche mit Tagesaufenthaltsmöglichkeiten (Wärmestuben, Suppenküchen, Essenstafeln), Übernachtungsangeboten (Heime, Pensionen, Wohnungen, Notschlafstellen), Gesundheitshilfen (Straßenambulanz) sowie tagesstrukturierenden Aktivitäten (Werkstätten, Kreativprojekte, Sport) zwar gut ausdifferenziert sind, aber der Heterogenität der Zielgruppe nicht mehr gerecht werden. So wird im wissenschaftlichen Diskurs darauf hingewiesen, dass nicht alle Wohnungslosen die Angebote annehmen. Dies wird beispielsweise damit erklärt, dass es sich beim System der Wohnungslosenhilfe um ein bürgerliches und paternalistisches System handelt, in dem sich Wohnungslose und Professionelle (z. B. Sozialarbeiter*innen) als Angehörige unterschiedlicher Milieus gegenüberstehen, die aufgrund der verschiedenen Normalitätsvorstellungen das Phänomen der Wohnungslosigkeit unterschiedlich interpretieren. Erst ein Verständnis der pluralen Lebenswelten von Wohnungslosen führt zu Angeboten, die den Bedarfen gerecht werden. Daher müssen die Partizipationsmöglichkeiten der Betroffenen ausgeweitet werden (Studeny 2020, 2015; Szynka 2014).

Obgleich in einer zunehmend digitalisierten Gesellschaft der Einsatz von digitalen Kommunikations- und Informationstechnologien immer selbstverständlicher wird, überrascht es, dass die Erhöhung der Teilhabechancen via digitalen Angeboten weder in der Forschung noch in der Praxis prominent thematisiert wird. Bisherige Forschungen zeigen zwar, dass viele wohnungslose Menschen Smartphones 
und Computer in ihrem täglichen Leben nutzen, auch wenn der Gebrauch innerhalb dieser Gruppe heterogen ist (Rhoades et al. 2017; Harris 2017; Humphry und Phil 2016; Humphry 2014; Guadagno et al. 2013; Eyrich-Garg 2011, 2010; Roberson und Nardi 2010; Le Dantec und Edwards 2008; Miller et al. 2005). Eine digitale Spaltung kann daher nicht zwischen wohnungslosen und nicht-wohnungslosen Menschen konstatiert werden - eher verläuft eine derartige Spaltung innerhalb der Gruppen. Allerdings sind die konkrete Nutzung digitaler Medien, die individuellen Bedarfe an digitaler Partizipation, die Auswirkung von Wohnungslosigkeit auf das Nutzungsverhalten und die Verbesserung von Teilhabechancen Wohnungsloser durch digitale Unterstützungsangebote bisher kaum erforscht.

\section{Forschungsziele, -design und methodisches Vorgehen}

Dieser bisher vernachlässigten Thematik geht das vom Bundesministerium für Bildung und Forschung (BMBF) für drei Jahre finanzierte Forschungsprojekt ,SIWo - Smart Inklusion für Wohnungslose" ${ }^{11}$ nach. Im Zentrum des Forschungsinteresses steht die Frage, welche Informations-, Unterstützungs- und Beratungsbedarfe bei wohnungslosen Menschen vorliegen und in welcher Form diese mit Hilfe von digitalisierten Angeboten gedeckt werden können. Mit diesem Fokus ist zu erwarten, dass neue wissenschaftliche Erkenntnisse über den Beitrag von digitalisierten Präventions-, Kooperations- und Interventionsmöglichkeiten zur gesellschaftlichen Inklusion von Wohnungslosen gewonnen werden. Gleichzeitig wird deutlich werden, wo Grenzen der Digitalisierung liegen.

Um die genannten Forschungsziele zu erreichen, wird ein qualitatives, partizipatives und interdisziplinäres Forschungsdesign gewählt. Hierfür werden zunächst die Bedarfe mit Hilfe von Gruppendiskussionen mit unterschiedlichen Stadtakteur*innen (z.B. Soziale Arbeit, Stadtverwaltung) und wohnungslosen Gruppen (z.B. jugendlichen Obdachlosen, wohnungslosen Frauen, Migrant*innen aus osteuropäischen EU-Staaten) sowie verstehenden leitfadengestützten Interviews mit Wohnungslosen erhoben. Der Samplingprozess orientiert sich dabei am forschungspraktischen Vorgehen der Grounded Theory (Glaser und Strauss 1998). Der zweistufige Auswertungsprozess sieht zunächst eine Erschließung der relevanten Kategorien des Feldes durch die Anwendung der Sequenzanalyse vor, bevor dann die Kodierung des gesamten empirischen Materials mit Verfahren der Grounded Theory erfolgt. Gleichzeitig vollzieht das Usability Engineering User Research mit ausgesuchten Expert*innengruppen (z.B. der Selbstvertretung wohnungsloser Menschen), bspw. Gruppendiskussionen, bei denen konkrete Themen gezielt gesetzt sind, um für im Vorfeld definierte Problemräume mit Hilfe der Expert*innen ein vertieftes Verständnis über die Kontexte, Erfordernisse, Probleme und Präferenzen von Nutzer*innen zu erhalten. Mit den Diskussionsergebnissen können (Benutzer*innen-)anforderun-

\footnotetext{
1 Das vom 01.08.2019 bis 31.07.2022 durchgeführte Forschungsprojekt wird vom Bundesministerium für Bildung und Forschung (BMBF) im Rahmen des Förderprogramms Forschung an Fachhochschulen gefördert (Förderkennzeichen: 13FH519SX7).
} 
gen identifiziert und in weiteren kollaborativen Prozessen Lösungsideen entwickelt werden (Geis et al. 2016).

Aus den gebündelten Ergebnissen der sozialwissenschaftlichen Forschung und des User Research werden Anforderungen für die Informatik und die Energietechnik abgeleitet und direkt in prototypische Lösungen in Form einer plattformübergreifenden Hilfe-App (webbasierte Applikationen für mobile und stationäre Endgeräte) überführt, welche in Nürnberg erprobt wird. Vorstellbar wäre, dass Informationen zum lokalen Hilfeangebot bereitgestellt und Formen von Onlineberatung sowie PeerBeratung bzw. ein Austausch unter Wohnungslosen ermöglicht werden. Im Mittelpunkt könnten interaktive Funktionen stehen, die dazu führen, dass die digitalisierten Angebote sich nicht nur auf die beschriebenen Zielgruppen konzentrieren, sondern alle Stadtakteur*innen beteiligen (z. B. Soziale Arbeit, Stadtverwaltung, Zivilgesellschaft/Bürgerschaft, lokale Wirtschaft wie Wohnungswirtschaft oder Gastronomie etc.) und auf diese Weise digitalisierte Begegnungsräume geschaffen und Interaktionen auch zwischen Bevölkerungsgruppen gefördert werden, deren Lebenswelten sonst getrennt sind. Smart-City Elemente könnten die Teilhabechancen von Wohnungslosen an der urbanen Infrastruktur verbessern, z. B. wenn die Zielgruppen internetfähige, robuste Smartphones, Zugang zu kostenfreiem WLAN und Lademöglichkeiten sowie Schulungen erhalten. In den Testphasen der Prototypen wird das tatsächliche Nutzungsverhalten der Adressat*innen mit Hilfe von teilnehmenden Beobachtungen erforscht und evaluiert, welche Gruppen von Wohnungslosen durch die digitalen Angebote erreicht werden und ob Teilhabechancen verbessert werden. Dabei wird eine Herausforderung des Projekts sein, den Schutz von sensiblen Daten zu gewährleisten.

Der verstehende Zugang zur Situation Wohnungsloser und sich in Wohnungsnot befindlicher Menschen, die Offenheit für bislang unbekannte Lösungsansätze, die Modularität des Servicedesigns und die Perspektiverweiterung auf gesamtgesellschaftliche Akteure spiegeln die Heterogenität der Wohnungslosen und die sich verändernden gesellschaftlichen Rahmenbedingungen wider.

Funding Open Access funding provided by Projekt DEAL.

Open Access Dieser Artikel wird unter der Creative Commons Namensnennung 4.0 International Lizenz veröffentlicht, welche die Nutzung, Vervielfältigung, Bearbeitung, Verbreitung und Wiedergabe in jeglichem Medium und Format erlaubt, sofern Sie den/die ursprünglichen Autor(en) und die Quelle ordnungsgemäß nennen, einen Link zur Creative Commons Lizenz beifügen und angeben, ob Änderungen vorgenommen wurden.

Die in diesem Artikel enthaltenen Bilder und sonstiges Drittmaterial unterliegen ebenfalls der genannten Creative Commons Lizenz, sofern sich aus der Abbildungslegende nichts anderes ergibt. Sofern das betreffende Material nicht unter der genannten Creative Commons Lizenz steht und die betreffende Handlung nicht nach gesetzlichen Vorschriften erlaubt ist, ist für die oben aufgeführten Weiterverwendungen des Materials die Einwilligung des jeweiligen Rechteinhabers einzuholen.

Weitere Details zur Lizenz entnehmen Sie bitte der Lizenzinformation auf http://creativecommons.org/ licenses/by/4.0/deed.de. 


\section{Literatur}

Eyrich-Garg, K. M. (2010). Mobile phone technology: a new paradigm for the prevention, treatment, and research of the non-sheltered "street" homeless? Journal of Urban Health, 87(3), 365-380.

Eyrich-Garg, K. M. (2011). Sheltered in cyberspace? Computer use among the unsheltered "street" homeless. Computers in Human Behavior, 27(1), 296-303.

Geis, T., Polkehn, K., Molich, R., \& Kluge, O. (2016). CPUX-UR Curriculum. Certified Professional for Usability and User Experience - User Requirements Engineering, Version 1.3. UXQB e. V. https:// uxqb.org/wp-content/uploads/documents/CPUX-UR_DE_Curriculum-und-Glossar.pdf. Zugegriffen: 3. März 2020.

Gerull, S. (2014). Wohnungslosigkeit in Deutschland. APuZ, 64(20-21), 30-36.

Glaser, B. G., \& Strauss, A. L. (1998). Grounded theory: Strategien qualitativer Forschung. Bern: Huber.

Guadagno, R.E., Muscanell, N.L., \& Pollio, D.E. (2013). The homeless use Facebook?! Similarities of social network use between college students and homeless young adults. Computers in Human Behavior, 29(1), 86-89.

Harris, J. (2017). The shift to digital advice and benefit services: implications for advice providers and their clients. In S. Kirwan (Hrsg.), Advising in austerity: Reflections on challenging times for advice agencies (S. 53-62). Bristol: Policy Press.

Holm, A. (2014). Mietenwahnsinn. Warum Wohnen immer teurer wird und wer davon profitiert. München: Knaur.

Humphry, J. (2014). Homeless and connected: mobile phones and the internet in the lives of homeless australians. Sydney: Australian Communications Consumer Action.

Humphry, J., \& Phil, K. (2016). Making connections. Young people, Homelessness and digital access in the city. Bd. 7. Abbotsford: Young and WEll CRC.

Le Dantec, C.A., \& Edwards, W. K. (2008). Designs on dignity: perceptions of technology among the homeless. Proceedings of the SIGCHI conference on human factors in computing systems.

Levinson, D. (2004). Encyclopedia of homelessness. London: SAGE.

Malyssek, J., \& Störch, K. (2009). Wohnungslose Menschen - Ausgrenzung und Stigmatisierung. Freiburg: Lambertus.

Miller, K. S., Bunch-Harrison, S., Brumbaugh, B., Kutty, R. S., \& FitzGerald, K. (2005). The meaning of computers to a group of men who are homeless. Department of occupational therapy faculty papers, paper 51 .

Paegelow, C. (2012). Handbuch Wohnungsnot und Obdachlosigkeit: Einführung in das Problemfeld der Wohnungslosen- und Obdachlosenhilfe. Bremen: Selbstverlag.

Paulgerg-Muschiol, L. (2009). Wege in die Wohnungslosigkeit. Eine qualitative Untersuchung. Siegen: Universität Siegen.

Rhoades, H., Wenzel, S. L., Rice, E., Winetrobe, H., \& Henwood, B. (2017). No digital divide? Technology use among homeless adults. Journal of Social Distress and the Homeless, 26(1), 73-77.

Roberson, J., \& Nardi, B. (2010). Needs and social inclusion: technology use among the homeless. Proceedings of the 2010 ACM conference on Computer supported cooperative work.

Sowa, F. (Hrsg.). (2020). Figurationen der Wohnungsnot. Kontinuität und Wandel sozialer Praktiken, Sinnzusammenhänge und Strukturen. Weinheim, Basel: Beltz Juventa.

Studeny, S. (2015). Internetcafé ZwischenSchritt. Überwindung der „Digital Gap“ im Rahmen der Wiener Wohnungslosenhilfe. Soziales Kapital, 13, 160-172.

Studeny, S. (2020). Digitalisierung in der Obdachlosenhilfe. In N. Kutscher, T. Ley, U. Seelmeyer, F. Siller, A. Tillmann \& I. Zorn (Hrsg.), Handbuch Soziale Arbeit und Digitalisierung (S. 552-564). Weinheim, Basel: Beltz Juventa.

Szynka, P. (2014). Partizipation in der Wohnungslosenhilfe. ARCHIV für Wissenschaft und Praxis der sozialen Arbeit, 2, 2-9.

Wehrheim, J. (2012). Die überwachte Stadt. Sicherheit und Ausgrenzung (3. Aufl.). Leverkusen: Budrich. 